JOURNAL OF THE

CHUNGCHEONG MATHEMATICAL SOCIETY

Volume 27, No. 2, May 2014

http://dx.doi.org/10.14403/jcms.2014.27.2.237

\title{
PRICING MULTI-ASSET DERIVATIVES WITH REGIME-SWITCHING VOLATILITIES
}

\author{
KUM-HWAN ROH*
}

\begin{abstract}
In this paper we provide a valuation method for multiasset derivatives with single jump regime-switching volatilities. We suppse that volatilities of assets are affected by an $n$-dimensional independent Markov regime-switching process.
\end{abstract}

\section{Introduction}

In Yoon et al.([6]), an analytic valuation method for multi-asset derivatives is investigated by using a Markov regime-switching volatility model. They assume that the volatilities of the assets have two states and the changes of the states are governed by a 1-dimensional continuous-time Markov chain. This assumption means that the volatilities of assets are influenced by one market environment.

By reason of the empirical importance in financial markets, the valuation methods of derivatives are widely studied with a regime-switching volatility model.([1], [2], [5], [7])

In this paper we assume that the volatilities of underlying assets are affected by an $n$-dimensional independent Markov regime-switching. This means that each volatility of asset is influenced by a separated independent market uncertainty. We use a single jump regime-switching process which is used in $\operatorname{Roh}([4])$, Zhang and Zhou([7]).

Received January 17, 2014; Accepted April 07, 2014.

2010 Mathematics Subject Classification: Primary 91G20.

Key words and phrases: regime-switching, multivariate contingent claim, pricing derivatives.

This work was supported by the 2014 Hannam University Research Fund. 


\section{The model}

We consider a filtered probablility space $\left(\Omega, \mathcal{F},\{\mathcal{F}\}_{t \geq 0}, P\right)$. The filtration $\{\mathcal{F}\}_{t \geq 0}$ satisfies the usual conditions and is generated by $n$ dimensional correlated Brownian motion $B(t)=\left(B_{1}(t), \cdots, B_{n}(t)\right)$ and $n$-dimensional independent Markov chain $\epsilon(t)=\left(\epsilon_{1}(t), \cdots, \epsilon_{n}(t)\right)$. And we suppose that Markov chains $\epsilon_{i}(t)$ are independent of each other and also independent of the Brownian motion $B(t)$. We denote the correlation between $B_{i}(t)$ and $B_{j}(t)$ as $\rho_{i j}$ for $i \neq j \in\{1, \cdots, n\}$. In order to ease of the calculations we define the $t$-forward price of $i$ th asset which is given by

$$
F_{i}(t)=S_{i}(0) \exp \left\{\int_{0}^{t}\left(r(s)-d_{i}(s)\right) d s\right\},
$$

where $r(\cdot)$ is interest rate and $d_{i}(\cdot)$ are dividend yields. Throughout the paper, we regard $r(\cdot)$ and $d_{i}(\cdot)$ as deterministic functions.

And we suppose that $n$ underlying assets prices are represented as

$$
S_{i}(t)=F_{i}(t) \exp \left\{-\int_{0}^{t} \frac{1}{2}\left(\sigma^{i}(s)\right)^{2} d s+\int_{0}^{t} \sigma^{i}(s) d B_{i}(s)\right\},
$$

where the $B_{i}(\cdot)$ are correlated one-dimensional Brownian motion with a constant correlation $\rho_{i j}$ between $B_{i}(\cdot)$ and $B_{j}(\cdot)$ and $i, j \in\{1, \cdots, n\}$.

We assume that the volatility $\sigma^{i}(t)$ is affected by a continous-time Markov chain $\epsilon_{i}(t)$. Also we assume that there are two regime states, $\operatorname{high}(\mathrm{H})$ and low $(\mathrm{L})$ states. The volatilities $\sigma^{i}(\cdot)$ can take a value in $\left\{\sigma_{H}^{i}, \sigma_{L}^{i}\right\}$. And regime $k$ switches into regime $l$ at the first jump time of an independent Poisson process with intensity $\lambda_{k}^{i}$, for $k, l \in\{H, L\}$. In other words, the random time $\tau_{k}^{i}$ of the leaving regime $k$ has an exponential distribution with intensity $\lambda_{k}^{i}$, i.e $P\left(\tau_{k}^{i}>t\right)=e^{-\lambda_{k}^{i} t}, k \in$ $\{H, L\}$.

It is assumed that a regime-switching model has a single jump over the life time of derivatives. Let the generator of a Markov chain $\epsilon_{i}(t)$ have the following form,

$$
\left(\begin{array}{cc}
-\lambda_{k}^{i} & \lambda_{k}^{i} \\
0 & 0
\end{array}\right)
$$

with a positive intensity $\lambda_{k}^{i}, k \in\{H, L\}$ and $i \in\{1, \cdots, n\}$. Then the $l$-state is the absorbing state. This model is called a single jump regimeswitching volatility model.

Proposition 2.1. Let $\sigma^{i}(s)$ be governed by a Markov chain $\epsilon_{i}(s)$ which have the generator, equation (2.2), where $i \in\{1, \cdots, n\}$. Then 
$\int_{0}^{t} \sigma^{i}(s) d B_{i}(s)$ is normally distributed with mean zero and variance $\left(\left(\sigma_{k}^{i}\right)^{2}-\left(\sigma_{l}^{i}\right)^{2}\right) \tau_{k}^{i}+\left(\sigma_{k}^{i}\right)^{2} t$, where $\sigma^{i}(0)=\sigma_{k}^{i}$ and $l \neq k \in\{H, L\}$.

Proof. From the assumption of a single jump regime-switching volatility model, the stochastic integral is derived as

$$
\int_{0}^{t} \sigma^{i}(s) d B_{i}(s)=\int_{0}^{\tau_{k}^{i}} \sigma_{k}^{i} d B_{i}(s)+\int_{\tau_{k}^{i}}^{t} \sigma_{l}^{i} d B_{i}(s),
$$

where $\tau_{k}^{i}$ is a random time of the leaving regime $k$.

Since $\sigma_{k}^{i}, \sigma_{l}^{i}$ are constants, the equation (2.3) is linear combination of Ito's integrals $\int_{0}^{\tau_{k}^{i}} d B_{i}(s)$ and $\int_{\tau_{k}^{i}}^{t} d B_{i}(s)$. Clearly, $\int_{0}^{\tau_{k}^{i}} d B_{i}(s)$ and $\int_{\tau_{k}^{i}}^{t} d B_{i}(s)$ are normally distributed with mean zero and variance $\tau_{k}^{i}, t-$ $\tau_{k}^{i}$, respectively. Also, two normal random vaiables $\int_{0}^{\tau_{k}^{i}} d B_{i}(s)$ and $\int_{\tau_{k}^{i}}^{t} d B_{i}(s)$ are independent. Hence the equation (2.3), the linear combination of $\int_{0}^{\tau_{k}^{i}} d B_{i}(s)$ and $\int_{\tau_{k}^{i}}^{t} d B_{i}(s)$ is normally distributed with mean zero and variance $\left(\left(\sigma_{k}^{i}\right)^{2}-\left(\sigma_{l}^{i}\right)^{2}\right) \tau_{k}^{i}+\left(\sigma_{k}^{i}\right)^{2} t$.

By using the Proposition 2.1 we can simplify the asset price process, the equation (2.1) as

$$
\begin{aligned}
S_{i}(t)=F_{i}(t) \exp & \left\{-\frac{1}{2}\left(\left(\left(\sigma_{k}^{i}\right)^{2}-\left(\sigma_{l}^{i}\right)^{2}\right) \tau_{k}^{i}+\left(\sigma_{l}^{i}\right)^{2} t\right)\right. \\
+\sqrt{\left(\left(\sigma_{k}^{i}\right)^{2}-\left(\sigma_{l}^{i}\right)^{2}\right) \tau_{k}^{i}+\left(\sigma_{k}^{i}\right)^{2} t} & \left.Y_{i}(t)\right\},
\end{aligned}
$$

where $Y_{i}(t)=\frac{\int_{0}^{t} d B_{i}(s)}{\sqrt{\left(\left(\sigma_{k}^{i}\right)^{2}-\left(\sigma_{l}^{i}\right)^{2}\right) \tau_{k}^{i}+\left(\sigma_{k}^{i}\right)^{2} t}}, i \in\{1, \cdots, n\}$ and $k, l \in$ $\{H, L\}$. Given $0 \leq \tau_{k}^{i}=s \leq t$, Propostion 2.1 tells us that $Y_{i}(\cdot)$ has a standard normal distribution.

Now we verify that an $n$-dimensonal random vector $Y(t)=\left(Y_{1}(t), \cdots\right.$, $\left.Y_{n}(t)\right)$ has a multivariate normal distribution from the next proposition.

Proposition 2.2. Let $\tau_{k_{i}}^{i}, \tau_{k_{j}}^{j}$ be the given jumping time which allow $\sigma_{k_{i}}^{i}$ and $\sigma_{k_{j}}^{j}$ to move $\sigma_{l_{i}}^{i}$ and $\sigma_{l_{j}}^{j}$, respectively. Then the random vector 
$Y(t)$ is a mulativaiate normal with mean 0 and covariances

$$
\operatorname{Cov}\left(Y_{i}(t), Y_{j}(t)\right)=\rho_{i j} \sigma_{k_{i}}^{i} \sigma_{k_{j}}^{j} \sqrt{\tau_{k_{i}}^{i} \tau_{k_{j}}^{j}}+\rho_{i j} \sigma_{l_{i}}^{i} \sigma_{l_{j}}^{j} \sqrt{\left(t-\tau_{k_{i}}^{i}\right)\left(t-\tau_{k_{j}}^{j}\right)},
$$

where $i, j \in\{1, \cdots, n\}, k_{i}, k_{j}, l_{i}, l_{j} \in\{H, L\}$ and $k_{i}, k_{j}$ are intial states of $\sigma^{i}(\cdot), \sigma^{j}(\cdot)$.

Proof. Let $\widetilde{B}(t)$ be an $n$-dimensional new Brownian motion which has mutually independent components. Then the random vector is decomposed as follows:

$$
\begin{aligned}
& \left(\int_{0}^{t} \sigma^{1}(s) d B_{1}(s), \cdots, \int_{0}^{t} \sigma^{n}(s) d B_{n}(s)\right)^{T} \\
& =\left(\begin{array}{ccc}
\sigma_{k_{1}}^{1} & \cdots & 0 \\
\vdots & \ddots & \vdots \\
0 & \cdots & \sigma_{k_{n}}^{n}
\end{array}\right) \cdot \Sigma^{1 / 2} \cdot\left(\begin{array}{c}
\int_{0}^{\tau^{1}} d \widetilde{B}_{1}(s) \\
\vdots \\
\int_{0}^{\tau^{n}} d \widetilde{B}_{n}(s)
\end{array}\right) \\
& +\left(\begin{array}{ccc}
\sigma_{l_{1}}^{1} & \cdots & 0 \\
\vdots & \ddots & \vdots \\
0 & \cdots & \sigma_{l_{n}}^{n}
\end{array}\right) \cdot \Sigma^{1 / 2} \cdot\left(\begin{array}{c}
\int_{\tau^{1}}^{t} d \widetilde{B}_{1}(s) \\
\vdots \\
\int_{\tau^{n}}^{t} d \widetilde{B}_{n}(s)
\end{array}\right),
\end{aligned}
$$

where $\Sigma$ is the variance-covariance matrix of $n$-dimensional Brownian motion $B(t)$.

Since Brownian motions $\widetilde{B}_{i}(t), \widetilde{B}_{j}(t)$ and random time processes $\tau^{i}, \tau^{j}$ are mutually independent for any $i \neq j \in\{1, \cdots, n\}$, the cross-variation $\left.\left\langle\int_{0}^{\cdot} d \widetilde{B}_{i}(s), \int_{0}^{\cdot} d \widetilde{B}_{j}(s)\right\rangle_{t}\right|_{\tau^{i}=\tau_{k_{i}}^{i}, \tau^{j}=\tau_{k_{j}}^{j}}=0$ for given jumping time $\tau_{k_{i}}^{i}, \tau_{k_{j}}^{j}$. From Knight Theorem([3]), $\left.\int_{0}^{\tau^{i}} d \widetilde{B}_{i}(s)\right|_{\tau^{i}=\tau_{k_{i}}^{i}}$ are mutually independent random variables, which have a normal distribution with mean zero and variance $\tau_{k_{i}}^{i}$. Similarly, we can show that $\left.\int_{\tau^{i}}^{t} d \widetilde{B}_{i}(s)\right|_{\tau^{i}=\tau_{k_{i}}^{i}}$ are mutually independently normally-distributed with mean zero and varaince $t-\tau_{k_{i}}^{i}$.

Hence the random vector $\left(\int_{0}^{t} \sigma^{1}(s) d B_{1}(s), \cdots, \int_{0}^{t} \sigma^{n}(s) d B_{n}(s)\right)^{T}$ has a multivariate normal distribution satisfying the conditions that the means of all components are zero, the variance of $i$ th component is $\left(\left(\sigma_{k}^{i}\right)^{2}-\left(\sigma_{l}^{i}\right)^{2}\right) \tau_{k}^{i}+\left(\sigma_{k}^{i}\right)^{2} t$ and the covariance of the $i$ th and $j$ th components is $\rho_{i j} \sigma_{k_{i}}^{i} \sigma_{k_{j}}^{j} \sqrt{\tau_{k_{i}}^{i} \tau_{k_{j}}^{j}}+\rho_{i j} \sigma_{l_{i}}^{i} \sigma_{l_{j}}^{j} \sqrt{\left(t-\tau_{k_{i}}^{i}\right)\left(t-\tau_{k_{j}}^{j}\right)}$. 
The value of a European multi-asset derivative, $V\left(T, S_{1}(T), \cdots, S_{n}(T)\right)$ with expiry $T$ is the expected present value of the payoff in the riskneutral measure. The following theorem gives the pricing of multi-asset derivatives with a single jump regime-switching model.

Theorem 2.3. Let $g\left(T, S_{1}(T), \cdots, S_{n}(T)\right)$ be the payoff of a European multi-asset derivative in initial states $k_{1}, \cdots, k_{n} \in\{H, L\}$. Then its present value $V\left(T, S_{1}(T), \cdots, S_{n}(T)\right)$ satisfies

$$
\begin{gathered}
V\left(T, S_{1}(T), \cdots, S_{n}(T)\right) \\
=e^{-r T} E\left[g\left(T, S_{1}(T), \cdots, S_{n}(T)\right) \mid \sigma^{1}(0)=\sigma_{k_{1}}^{1}, \cdots, \sigma^{n}(0)=\sigma_{k_{n}}^{n}\right] \\
=e^{-r T} \int_{0}^{T} \cdots \int_{0}^{T} E\left[g\left(T, S_{1}(T), \cdots, S_{n}(T)\right) \mid \tau_{k_{1}}^{1}=s_{1}, \cdots, \tau_{k_{n}}^{n}=s_{n}\right] \\
\quad \times e^{-\lambda_{k_{1}}^{1} s_{1}} \cdots e^{-\lambda_{k_{n}}^{n} s_{n}} d s_{1} \cdots d s_{n}
\end{gathered}
$$

Proof. Since the property of conditional expectation, the expected value of the payoff is converted as follows:

$$
\begin{gathered}
E\left[g\left(T, S_{1}(T), \cdots, S_{n}(T)\right)\right] \\
\left.=E\left[\cdots E\left[g\left(T, S_{1}(T), \cdots, S_{n}(T)\right) \mid \tau_{k_{1}}^{1}=s_{1}, \cdots, \tau_{k_{n}}^{n}=s_{n}\right] \mid \tau_{k_{1}}^{1}=s_{1}\right] \cdots \mid \tau_{k_{n}}^{n}=s_{n}\right] \\
=\int_{0}^{T} \cdots \int_{0}^{T} E\left[g\left(T, S_{1}(T), \cdots, S_{n}(T)\right) \mid \tau_{k_{1}}^{1}=s_{1}, \cdots, \tau_{k_{n}}^{n}=s_{n}\right] \\
\times e^{-\lambda_{k_{1}}^{1} s_{1}} \cdots e^{-\lambda_{k_{n}}^{n} s_{n}} d s_{1} \cdots d s_{n}
\end{gathered}
$$

Since the formula of Theroem 2.3 contains a conditional expectation $E\left[g\left(T, S_{1}(T), \cdots, S_{n}(T)\right) \mid \tau_{k_{1}}^{1}=s_{1}, \cdots, \tau_{k_{n}}^{n}=s_{n}\right]$, we can get a closedform valuation of multi-asset derivatives only if we know the value of conditional expectation for given $s_{i}$. By Propositions 2.1 and 2.2, we can apply the results of constant volatility model to our regime-switching volatility model. For some derivatives, there is no closed form valuation such as the formula of Thoerem 2.3, since it is essentially a multiple integral problem. But it can be calculated using a various numerical approximation scheme.

\section{Conclusion}

In this paper, we have valued a multi-asset derivative when the volatilities of the asset price processes are modeled as single jump regimeswitching processes with two states. Unlike the studies made extensively 
on the regime-switching, very little attention was given to the relation of regime change in assets.

We assume that the each volatility of asset price process could change the regime state, independently. In a financial market, underlying assets of multi-asset derivatives can be in the seperated market. This could cause that the regime-switching of environment does not influence the other environment. In this regime-switching market environment we provide a valuation method for multi-asset derivatives.

\section{References}

[1] C. D. Fhu, I. Hu, and S. K. Lin, Empirical performance and asset pricing in hidden Markov models, Commun. Statist.: Theory Math. 13 (2003), 2477-2512.

[2] A. T. Hansen and R. Poulsen, A simple regime switching term structure model, Fin. Stoch. 4 (2000), 409-429.

[3] I. Karatzas and S. E. Shreve, Brownian motion and stochastic calculus, Springer, 2000.

[4] K. Roh, Pricing commodity futures contracts with a regime-switching model, J. Chungcheong Math. 26 (2013), 787-791.

[5] K. Roh, Variance swap pricing with a regime-switching market environment, Manage. Sci. and Fin. Eng. 19 (2013), 49-52.

[6] J. H. Yoon, B. Jang, and K. Roh, An analytic valuation method for multivariate contingent claims with regime-switching volatilities, Oper. Res. Letters. 39 (2011), 180-187.

[7] Q. Zhang and X. Y. Zhou, Valuation of stock loans with regime-switching, SIAM J. Control and Optim. 48 (2009), 1229-1250.

Department of Mathematics

Hannam University

Daejeon 306-791, Republic of Korea

E-mail: khroh@hnu.kr 